\title{
Phase-Locked-Loop Adaptive-Optic Controller and Simulated Shear Layer Correction
}

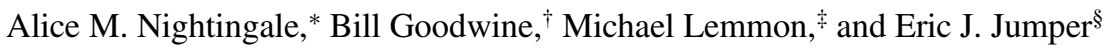 \\ University of Notre Dame, Notre Dame, Indiana 46556
}

\begin{abstract}
DOI: $10.2514 / 1 . J 052425$
An alternative adaptive-optic controller, using both flow control and a phase-lock-loop control strategy, has been designed to overcome bandwidth limitations inhibiting current adaptive-optic controllers. A discrete-vortex code and weakly compressible model were used to simulate high-speed shear layer adaptive-optic corrections based upon the proposed phase-lock-loop controller given a range of upper and lower Mach numbers. The shear layer was forced at its origin, creating a region of regularized large-scale structures through which a simulated optical beam was projected. The controller applied a predicted conjugate correction to the shear layer's emerging wavefront in a feedforward approach. The phase-lock-loop controller produces a sinusoidal signal for which the amplitude and phase are adjusted in real time to synchronize with the reference input. The controller is designed to track abrupt changes in phase or frequency. An overview of the design process is provided along with the alternative adaptive-optic controller's basic layout and circuitry diagrams. Finally, experimental jitter results illustrate the controller's amplitude and phase response capabilities given a purely sinusoidal function generator input signal.
\end{abstract}

\section{Introduction}

$\mathbf{T}$ HE performance of optical systems, including free-space communication platforms and airborne laser systems, is dependent upon the ability to maintain the optical beam's integrity as it travels along its intended path. Index-of-refraction variations encountered by the beam, both in the near field and far field, impose aberrations, or unwanted curvature, to an otherwise planar optical wavefront. The ideal planar wavefront creates a far-field pattern known as the airy disk (shown in Fig. 1) with its maximum achievable intensity on axis (central location of far field). When an optical wavefront becomes aberrated, the far-field intensity pattern may be severely degraded, as shown by the example in Fig. 2 given a $\pm 1 \mu \mathrm{m}$ peak-to-peak sinusoidal aberration whose $\mathrm{OPD}_{\mathrm{rms}}$ is approximately $0.7 \mu \mathrm{m}$. Therefore, such aberrations require some type of correction in order to improve system performance. Such aberrations may be quantified using optical path difference (OPD) which is the integrated effect of a beam propagating through a variable index-ofrefraction field.

Adaptive optics, the technique of applying real-time conjugate corrections to an optical wavefront before its transmission through an aberrating medium effectively restoring a planar wavefront (Fig. 3), was first introduced by Horace Babcock in 1953 [1,2]. Since that time, several advancements have led to the development of feedback control systems used to perform real-time imaging corrections for low-frequency disturbances. These current adaptive-optic (AO) systems are being used to successfully correct wavefront aberrations induced by atmospheric turbulence occurring in the far field [1-4] . While current AO systems do perform well given low-frequency disturbances, they face significant limitations at higher frequencies.

Presented as Paper 2006-4211 at the 39th Plasmadynamics and Lasers Conference, San Francisco, California, 23-26 June 2008; received 3 November 2012; revision received 13 May 2013; accepted for publication 24 May 2013; published online 23 September 2013. Copyright (C) 2013 by Nightingale, A. M., Goodwine, B., Lemmon, M., and Jumper, E. J. Published by the American Institute of Aeronautics and Astronautics, Inc., with permission. Copies of this paper may be made for personal or internal use, on condition that the copier pay the $\$ 10.00$ per-copy fee to the Copyright Clearance Center, Inc., 222 Rosewood Drive, Danvers, MA 01923; include the code $1533-385 X / 13$ and $\$ 10.00$ in correspondence with the CCC.

*Research Assistant Faculty, Department of Mechanical and Aerospace Engineering, Hessert Laboratory for Aerospace Research.

${ }^{\dagger}$ Associate Professor, Department of Mechanical and Aerospace Engineering.

FProfessor, Department of Electrical Engineering.

${ }^{\S}$ Professor, Department of Mechanical and Aerospace Engineering, Hessert Laboratory for Aerospace Research. Fellow AIAA.
In the case of airborne laser systems, such as the turret/fairing illustration shown in Fig. 4, the optical signal must propagate through variable density flowfields containing frequencies that approach, and in some cases exceed, $1 \mathrm{kHz}$ [5-8]. Within the system's field of regard, the beam may encounter several different types of turbulence, one of which is a free shear layer. The high-frequency content and unpredictable nature of this type of flow severely degrades beam quality, adversely affecting system performance. And at present, the resulting high-frequency aberrations imposed on the optical beam as it propagates through a free shear layer cannot be successfully mitigated using the conventional AO approach due to bandwidth limitations caused by system gain requirements and latencies $[1,9,10]$. As such, research efforts over the past several years at the University of Notre Dame have been investigating an alternative approach to performing $\mathrm{AO}$ corrections to a beam experiencing highfrequency disturbances caused by a free shear layer [9-11].

\section{Alternative AO Approach}

Due to bandwidth limitations faced by current AO systems, an alternative approach was devised to handle high-speed $\mathrm{AO}$ corrections induced by a separated free shear layer. This approach comprises two separate components or types of control: the first is to use a form of flow control, such as mechanical forcing, to create a region of more regularized structures and induced aberrations; and the second is to use a phase-lock-loop (PLL) controller to synchronize a correction signal with a reference signal emerging from the regularized region of the shear layer. Once the signals become locked in phase, a priori knowledge of the large-scale structures convecting downstream within the forced shear layer is used to construct a two-dimensional AO correction to be applied to a deformable mirror.

\section{A. Shear Layer Regularization}

The alternative AO controller design, simulations, and preliminary experimental testing documented in this paper are the result of several years of research conducted at the University of Notre Dame [7,911]. It has long been known that large-scale structures that naturally "roll up" are the primary cause of optical aberrations in free shear layers [12-14]. However, more recently, it was found that the cause of the induced aberrations relates more specifically to the radial pressure gradients and associated density deficit required to support the curvature of these structures [15]. This study showed that the density deficit supporting the large-scale coherent structures, along with the high-pressure/density regions between rollers, gives rise to the majority of the optical aberration imparted on a beam propagating 

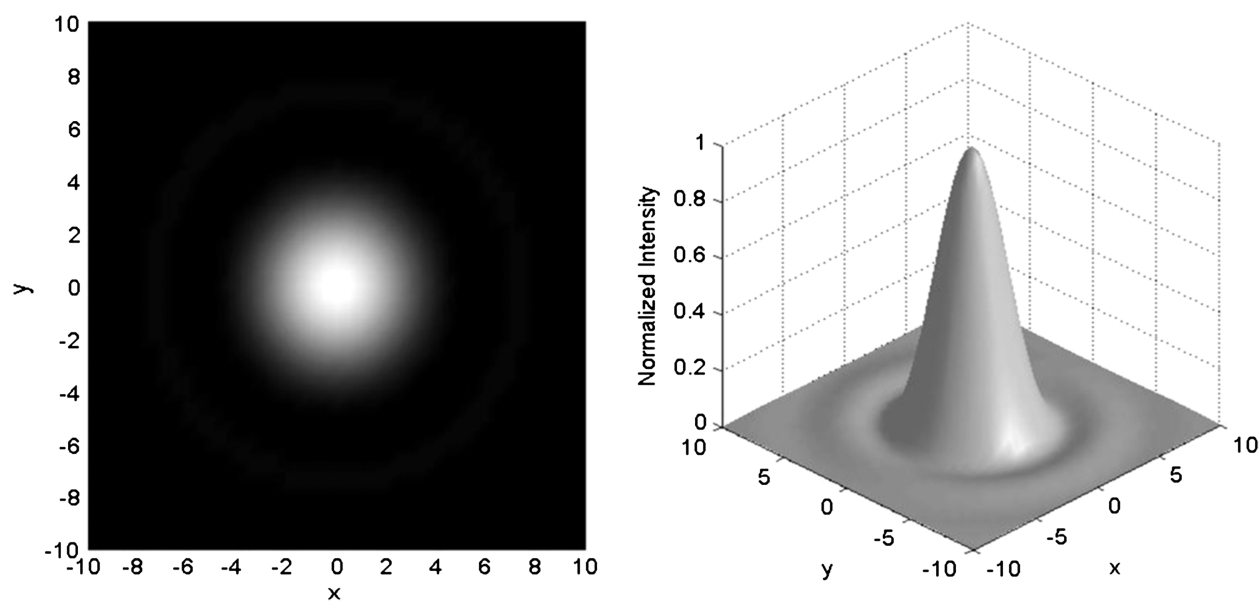

Fig. 1 Two- and three-dimensional simulated diffraction pattern for circular aperture, planar wavefront.
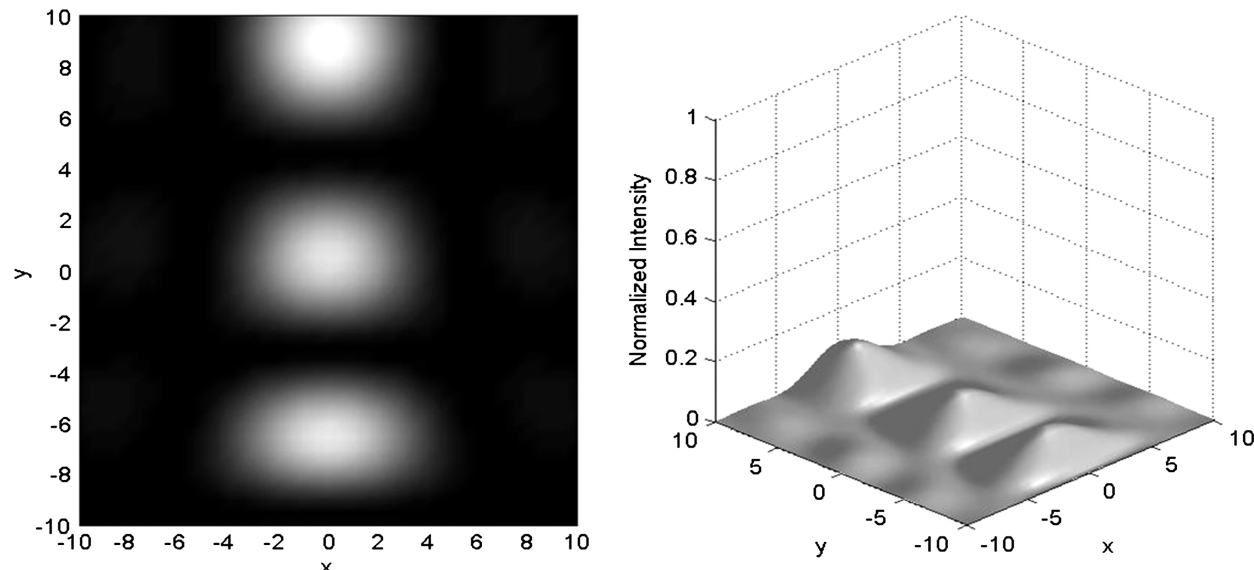

Fig. 2 Two- and three-dimensional simulated diffraction pattern given a sinusoidal wavefront aberration.
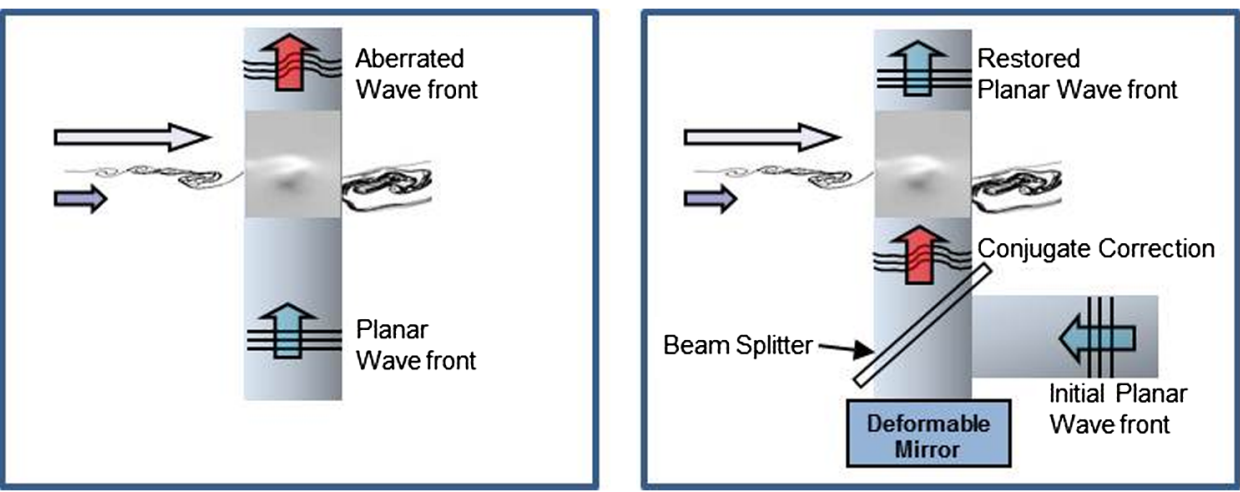

Fig. 3 Depiction of optical aberration emerging from shear layer with and without correction.

through the flow. The formation and evolution of large-scale structures were also known to be susceptible to forcing in mixing layers $[16,17]$. However, the ability to regularize large-scale structures within a high-speed shear layer remained in question. Furthermore, it was uncertain whether regularizing the shear layer's large-scale structures would in turn create a more regular, and consequently more periodic, emerging wavefront. As a result, a numerical investigation [9], followed by high-speed shear layer wind-tunnel experiments $[10,11]$, was conducted at the University of Notre Dame. These studies showed that regularizing the large-scale structures in a highspeed shear layer, along with their associated optical wavefronts, was indeed possible. In the numerical results published in 2009 by Nightingale et al. [9], high-speed shear layers were shown to contain a range of "natural optical" frequencies that, when excited, produced a region of regularized (more periodic in nature) large-scale structures. The location of this regularized region depended upon the downstream position where the shear layer's unforced natural optical frequency matched the forcing frequency. High-speed forced shear layer experiments conducted at the University of Notre Dame demonstrated the ability to regularize a region of large-scale structures through mechanical forcing at its origin [11]. Subsequently, Rennie et al. were able to successfully show that significant AO corrections could be achieved by applying a feedforward predictive waveform to a forced two-dimensional weakly compressible, shear layer once the corrective waveform was manually synchronized with the regularized large-scale structures [10]. This paper outlines the 


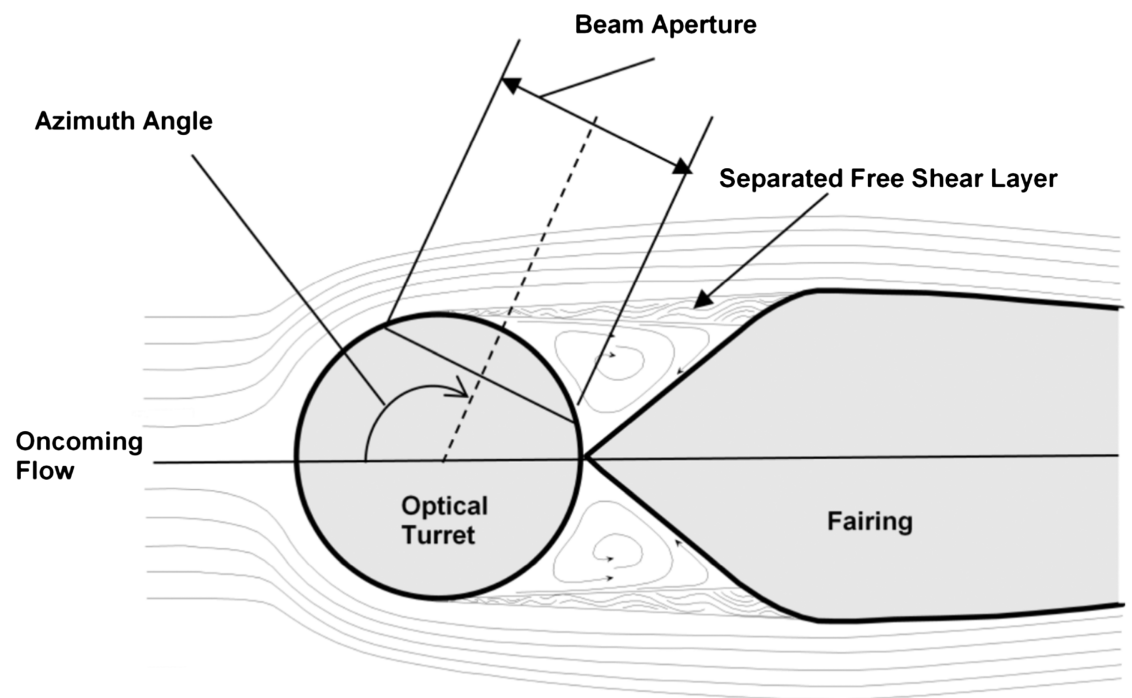

Fig. 4 Illustration of a shear layer formed over a turret/fairing combination.

design, construction, and preliminary experimental testing for a PLL AO controller used to automate the synchronization process referred to previously in which a predictive conjugate correction was synchronized with the forced shear layer's aberrating wavefront.

\section{B. Phase-Lock-Loop Control Technique}

Phase-lock-loops are a common control system used to synchronize the output signal with an input reference signal [18]. An example analogous to the PLL process is the use of a tuning fork to tune an instrument, where the tuning fork provides the reference input or standard of pitch. When the desired note is struck on the instrument, an audible beat frequency may be heard until the instrument is tuned so that its frequency matches that of the tuning fork. Similarly, when the PLL's output frequency is out of phase with respect to the reference, an error signal is produced. This signal is used to adjust the PLL's output frequency until the two signals become phase-locked and the error approaches zero.

A typical PLL comprises a phase detector, a loop filter, and a voltage-controlled oscillator (VCO), as illustrated in Fig. 5. As indicated by its name, the phase detector determines the phase difference between the output signal and the reference signal. For the controller outlined in this paper, this is accomplished using a simple multiplication chip in conjunction with a low-pass filter. The PLL's output signal is multiplied by a reference signal, creating an output at two different frequencies; a baseband portion contains the error estimate necessary for phase-locking and a double harmonic portion (twice the PLL operating frequency) that is filtered out. The loop filter for this application, designed by the control engineer to meet a desired system response, includes two poles at the origin of the complex plane (integrators) and a minimum phase zero. The double integral action allows the control system to respond to both step and ramp changes in phase with a zero tracking error, while the minimum phase zero is necessary to ensure closed-loop system stability. Finally, the VCO closes the loop by producing a sinusoidal output signal at a given center operating frequency. Acting as an integrator, the VCO's output frequency is adjusted based upon the loop filter's outgoing error signal, which serves as its dc input. The following section describes the process of designing the PLL AO controller: specifically, the loop filter portion.

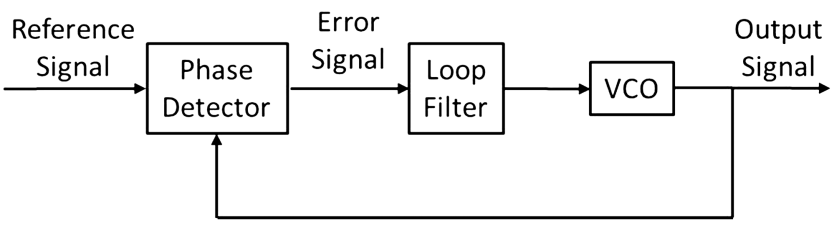

Fig. 5 Block diagram of the basic PLL process.

\section{PLL AO Controller Design}

To apply linear control techniques to the PLL, a more conceptual model must be used. Figure 6 shows the block diagram for the conceptual, linearized, PLL model in the phase domain. As shown, a summation block represents the mixing function of the phase detector. The output signal $Y(s)$ is subtracted from the reference signal $R(s)$, generating an error signal $E(s)$. The error is scaled by $K_{\mathrm{PD}}$, representing the gain magnitude of the phase detector, and passed through a low-pass filter $F(s)$. The loop filter $L_{F}(s)$ performs any other necessary filtering action while maintaining the system's closed-loop stability. Finally, the VCO acts as an integrator, where $K_{\mathrm{VCO}}$ is referred to as the sensitivity constant related to the specific VCO device.

The closed-loop transfer function for the system shown in Fig. 6 is given by the Laplace transform

$$
T(s)=\frac{Y(s)}{R(s)}=\frac{K_{\mathrm{PD}} K_{\mathrm{VCO}} F(s) L_{F}(s)}{s+K_{\mathrm{PD}} K_{\mathrm{VCO}} F(s) L_{F}(s)}
$$

where $F(s)$ represents the low-pass filter function, and $L_{F}(s)$ represents the loop filter function designed to meet the desired system response characteristics. For the aero-optic application referred to in this paper, the phase of the shear layer's emerging regularized wavefront serves as the reference source $R(s)$, and the phase of the wavefront estimation model used to control the deformable mirror represents the output, or phase-locking signal, $Y(s)$.

An electronic analog PLL circuit was designed and constructed for this research with the control objective of synchronizing itself with a regularized shear layer jitter signal for which the frequency ranges between approximately 600 and $1100 \mathrm{~Hz}$, centered around $850 \mathrm{~Hz}$. A low-pass filter with a cutoff frequency of approximately $80 \mathrm{~Hz}$ ( $\sim 500 \mathrm{rad} / \mathrm{s})$ was used to attenuate the double harmonic signal to approximately $0.2 \%$ of its original amplitude. The purpose of this filter is to isolate the dc component of the phase detectors outgoing mixing signal. The remaining gain and pole/zero placements were selected based upon five design criteria, including tracking error, phase margin, settling time, percent overshoot, and absolute error. To create a PLL capable of tracking both step and ramp phase changes with zero steady-state error, the filter function must contain two poles at the origin of the complex plane. As a result, a minimum phase zero

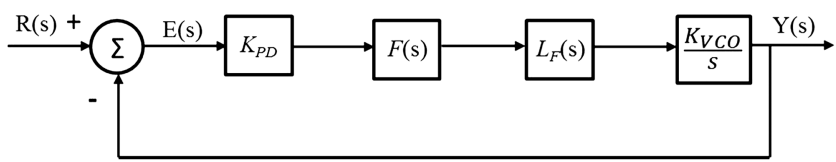

Fig. 6 Block diagram of a conceptual, linearized phase domain model for the basic PLL. 
must be included in the PLL design to shift the unity gain such that the phase margin remains positive, ensuring closed-loop stability.

Phase margin, settling time, percent overshoot, and two forms of absolute error were each plotted versus the placement of the loop filter's minimum phase zero for a range of overall gain constants. The design goal was to maximize phase margin (increasing phase margin improves the system's safety margin for stability) and minimize integral error while reducing the settling time and percent overshoot. Two forms of integral error were analyzed for this study: integral of time multiplied by absolute error (ITAE) and integral of the square of the error (ISE), defined, respectively, as

$$
\operatorname{ITAE}=\int_{0}^{T} t|e(r)| \mathrm{d} t
$$

and

$$
\mathrm{ISE}=\int_{0}^{T} e^{2}(t) \mathrm{d} t
$$

Figures 7-11 show the results for this set of design criteria. The graphs were studied collectively to determine the minimum phase

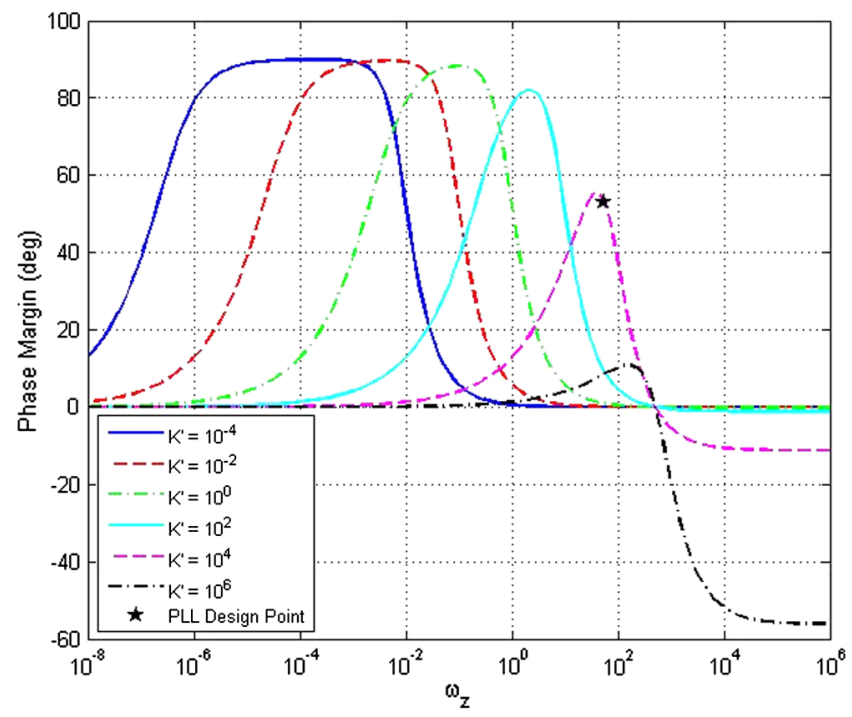

Fig. 7 Phase margin versus minimum phase zero $\left(\omega_{z}\right)$ for a range of gain constants $\left(K^{\prime}\right)$. zero and total gain constant combination appropriate for this controller. The primary goal was to maintain system stability while reducing the amount of integral error amassed throughout the AO correction process.

After evaluating the system response characteristics shown previously, an overall gain constant $K^{\prime}$ of approximately 10,000 was selected along with a minimum phase zero frequency $\omega_{z}$ of approximately $55 \mathrm{rad} / \mathrm{s}$. The final open-loop controller transfer function is given by

$$
G(s) H(s)=G_{C}(s)=\frac{5,000,000(s+55)}{55 s^{2}(s+500)}
$$

where a low-pass filter cutoff frequency of approximately $500 \mathrm{rad} / \mathrm{s}$ was applied. These design selections result in a phase margin of approximately $53 \mathrm{deg}$ at $179 \mathrm{rad} / \mathrm{s}$, a percent overshoot less than $25 \%$ given a step input and an ITAE index and ISE index below 0.0003 and 0.004 , respectively. Step and ramp responses based on this controller [Eq. (4)] were simulated to verify its response. As shown in Fig. 12, the output settles to within 1 and $0.01 \%$ of their final value in less than $0.05 \mathrm{~s}$ given a unit step input and ramp input, respectively.

\section{AO Simulations}

The finalized PLL AO controller was modeled and simulated in MATLAB $\triangle$ based on the design criteria outlined in the previous section. These simulations were conducted to evaluate the controller's response and effectiveness when used in a shear layer application. A discrete-vortex method (DVM) and weakly compressible model (WCM) [15] were used to simulate a free shear layer and its emerging optical wavefront. The DVM models a shear layer using two semi-infinite vortex sheets. The free vortices within a computational window are allowed to convect and move based on the induced velocity of neighboring vortices and the overall convection velocity of the flow. Forcing is applied at the shear layer's origin (splitter plate) by displacing a single vortex vertically (similar to mechanical forcing) in order to perturb the shear layer at a "natural optical frequency" [9], creating a region of regularized large-scale structures. Velocity fields are computed based on the discrete vortices positions after iterating the code through the transient phase. Thermodynamic properties of the flow are then overlain using the WCM given the DVM's time-evolving velocity fields. A onedimensional beam of a given aperture (or width) is numerically propagated through the WCM's simulated index-of-refraction field to produce time-varying one-dimensional optical wavefronts. The

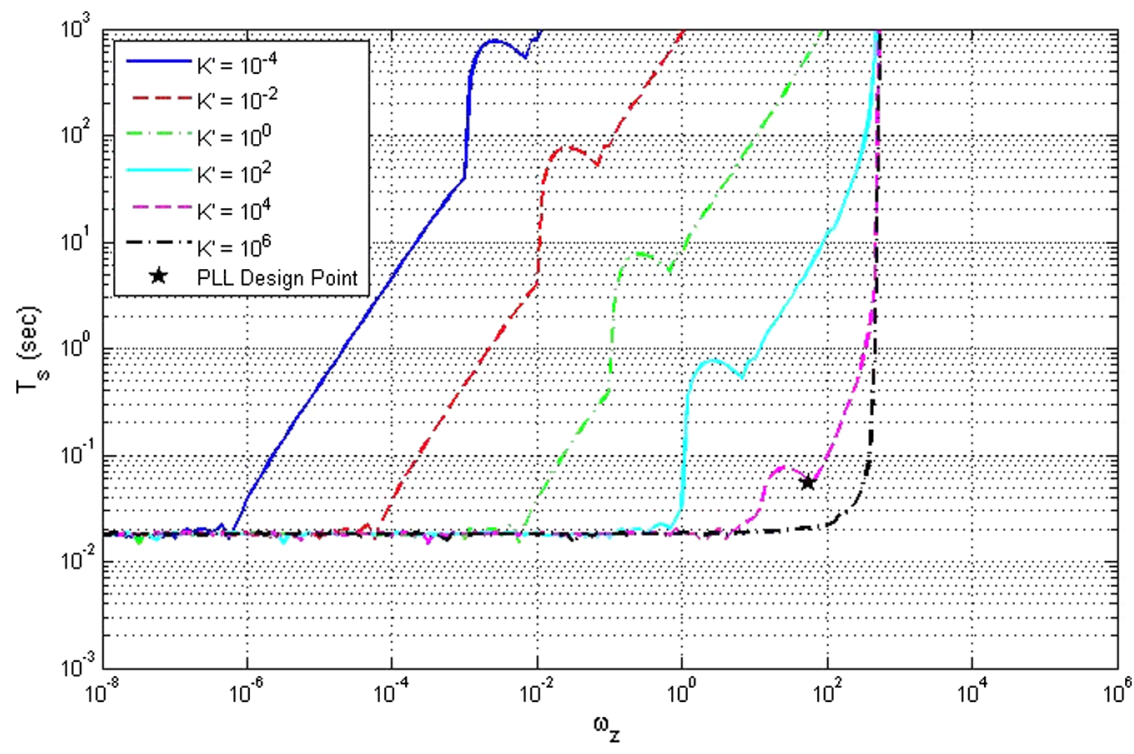

Fig. 8 Settling time $\left(T_{s}\right)$ versus minimum phase zero $\left(\omega_{z}\right)$ for a range of gain constants $\left(K^{\prime}\right)$ given a step input. 


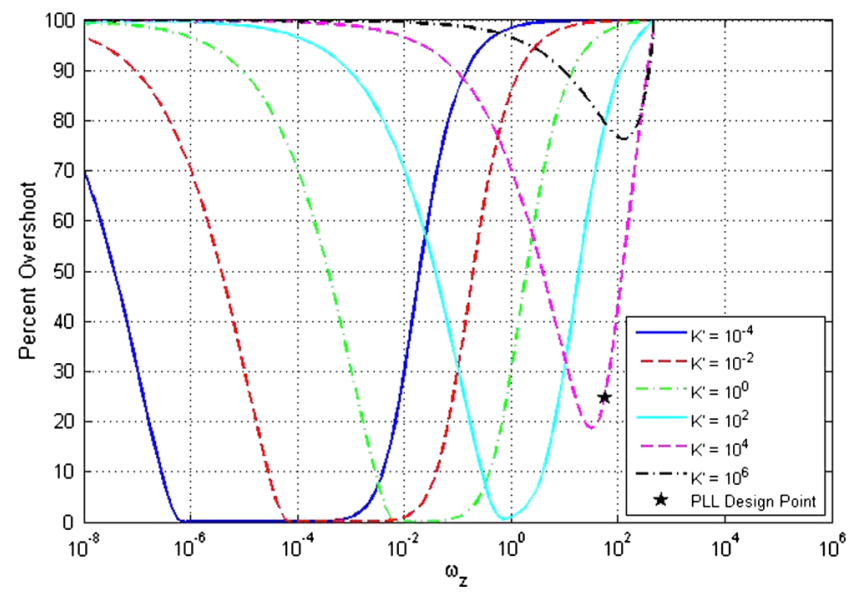

Fig. 9 Percent overshoot versus minimum phase zero $\left(\omega_{z}\right)$ for a range of gain constants $\left(K^{\prime}\right)$.

PLL's predictive correction is then subtracted from the DVM/WCM shear layer's emerging wavefront, simulating an adaptive-optic correction. Figure 13 shows a schematic of the described shear layer adaptive-optic correction simulation.

Three different simulated high-speed shear layer cases are reported in this section, for which the upper Mach numbers range from approximately 0.55 to 0.8 and lower Mach numbers range from approximately 0.1 to 0.2 . A small aperture beam was directed through the shear layer's regularized region numerically and used as the AO controller's reference input signal. While these tests were not experimental, the WCM's ability to predict the large-scale structures within the flowfield $[15,19,20]$ provides a good means of initially assessing the alternative $\mathrm{AO}$ control approach described in this paper.

A succession of optical wavefronts and a time series of Strehl ratios were computed before forcing to provide a reference with which to compare the correction results. The simulated shear layer was then forced, and AO corrections were applied using the simulated PLL controller described in Eq. (4). The first simulated shear layer study had upper and lower Mach numbers of approximately 0.55 and 0.17 , respectively, and was forced at $1100 \mathrm{~Hz}$. A 0.15 -m-aperture beam was constructed numerically from wavefront data centered approximately $0.38 \mathrm{~m}$ downstream from the shear layer's origin. Six consecutive numerical wavefronts simulated approximately $0.00015 \mathrm{~s}$ apart (where $\Delta$ corresponds to a 60 deg phase lag with respect to the forcing frequency) were computed both before (Fig. 14)

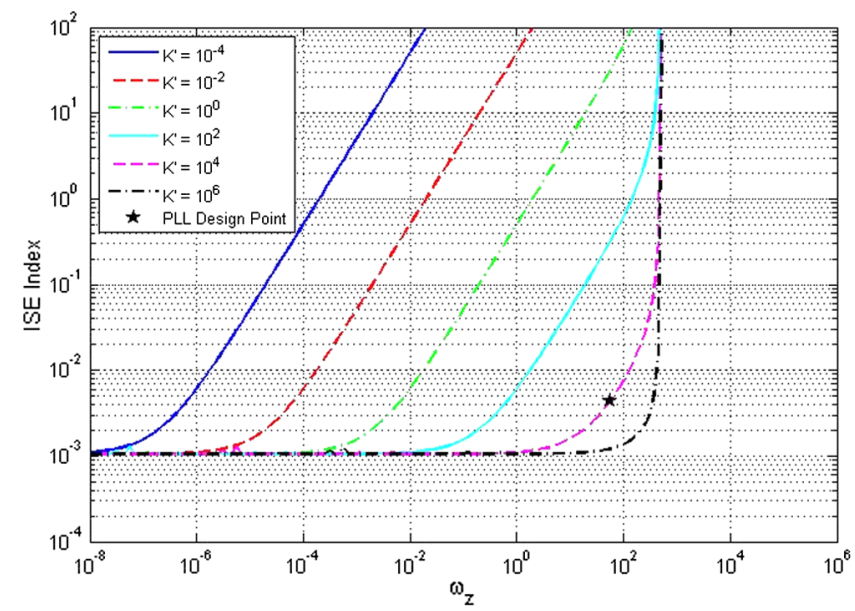

Fig. 11 ISE performance criterion versus minimum phase zero $\left(\omega_{z}\right)$ for a range of gain constants $\left(K^{\prime}\right)$.
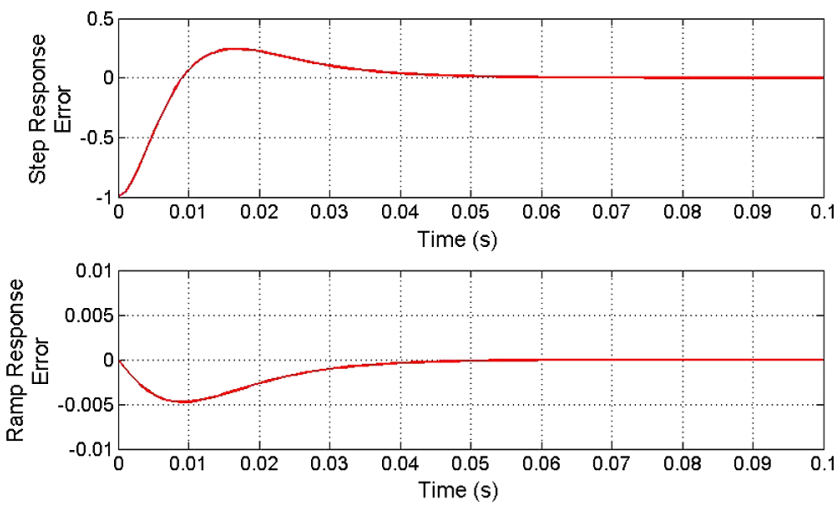

Fig. 12 PLL controller's simulated step response (upper plot) and ramp response (lower plot).

and after (Fig. 15) AO corrections were applied. In this case, the timeaveraged $\mathrm{OP} \overline{\mathrm{D}_{\mathrm{rms}}}$ error reduces from approximately 0.037 to $0.021 \mu \mathrm{m}$. It should be noted that the error may be reduced even further by removing tip/tilt (see later Strehl ratio results).

Strehl ratios were also computed for each numerical simulation before and after $\mathrm{AO}$ corrections were applied. Figure 16 shows the

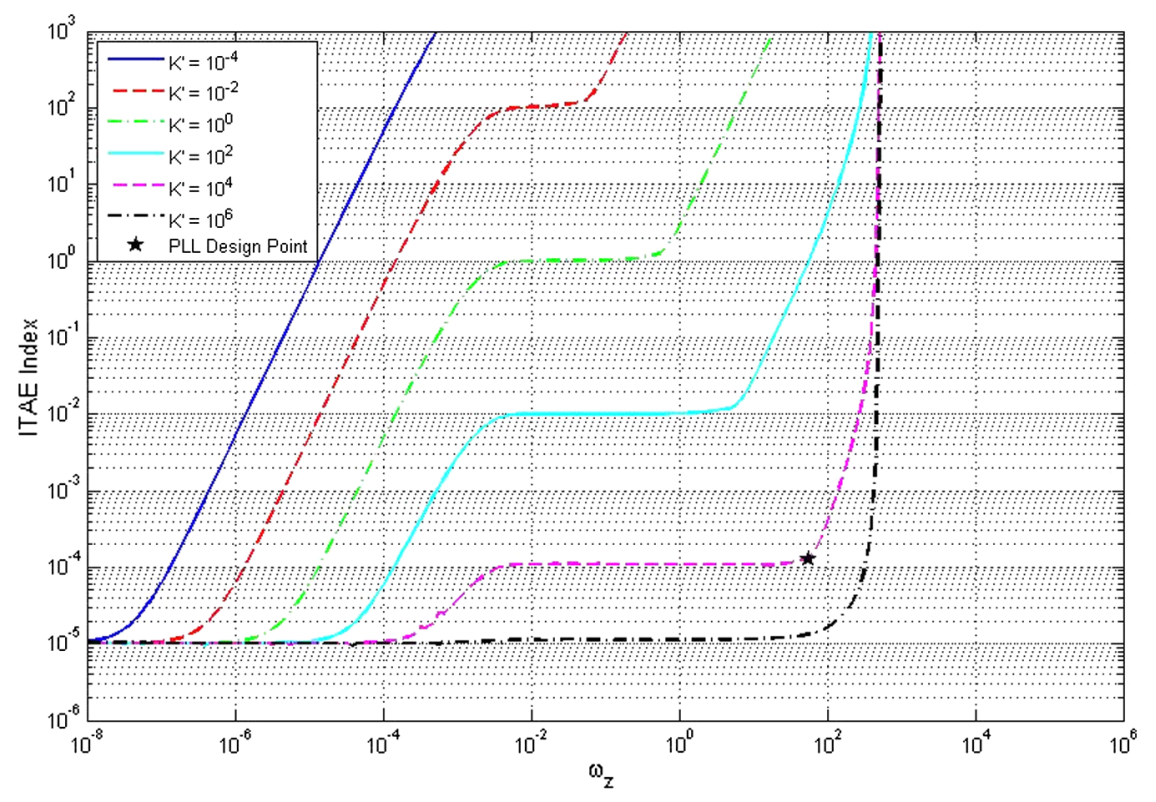

Fig. 10 ITAE performance criterion versus minimum phase zero $\left(\omega_{z}\right)$ for a range of gain constants $\left(K^{\prime}\right)$. 


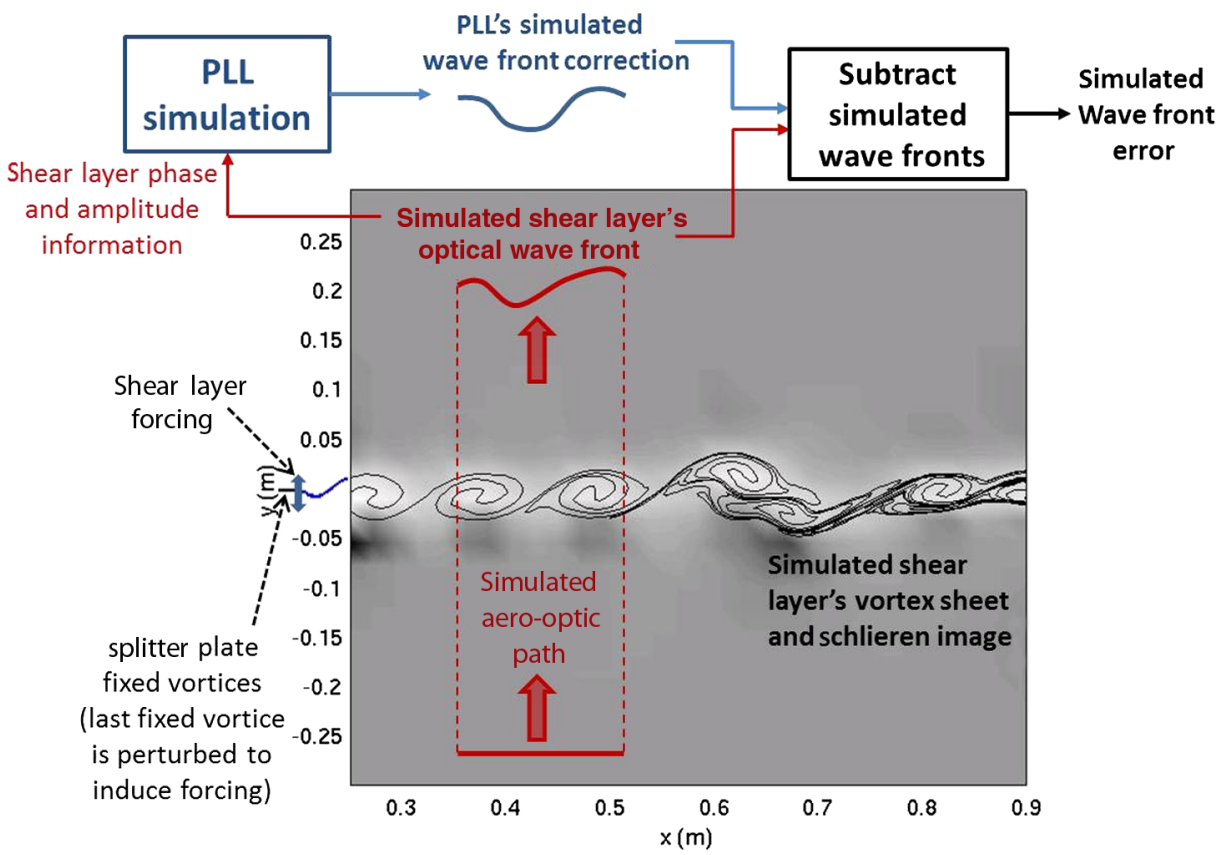

Fig. 13 Schematic of the shear layer adaptive-optic correction simulation.
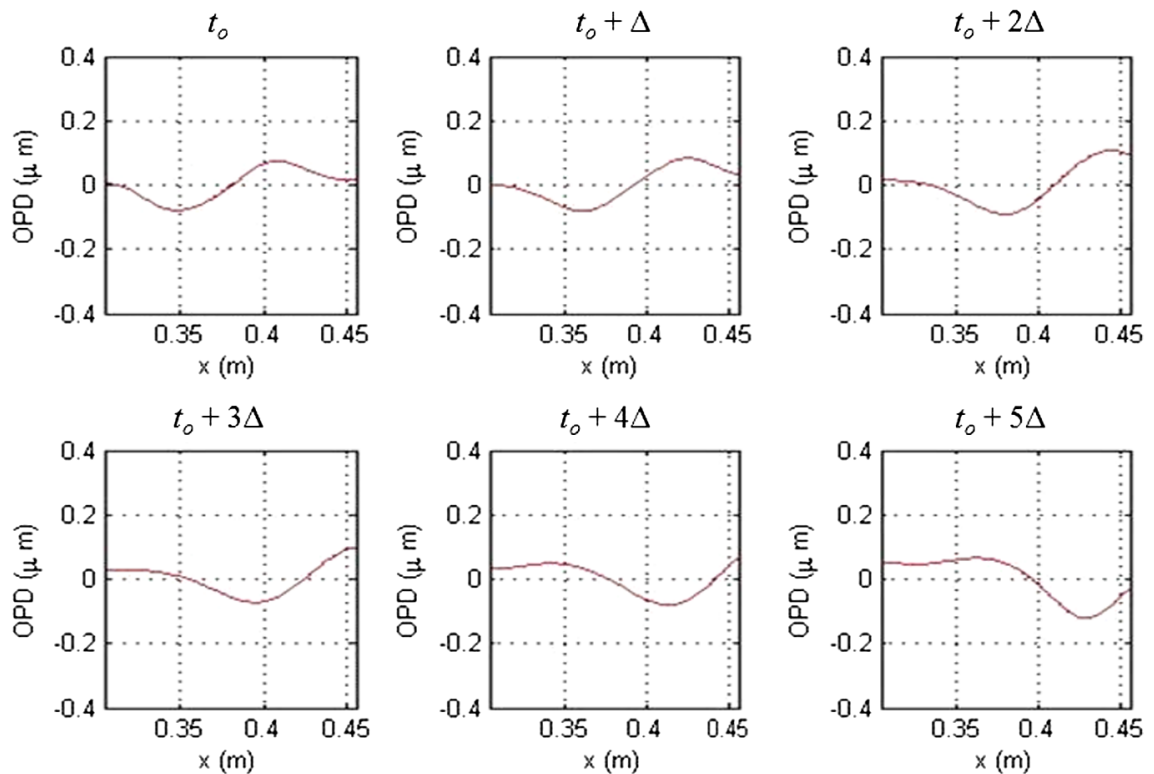

Fig. 14 Successive numerical wavefronts for a Mach 0.55/0.17 unforced shear layer.

Strehl ratio versus time for the unforced shear layer before making AO corrections. The time-averaged Strehl ratio for the given duration of time is approximately 0.85 . While this value is fairly high, it is clear from the graph that the instantaneous value frequently drops below 0.7. After regularizing a region of the flow through forcing and applying AO corrections using the simulation PLL controller, the time-averaged Strehl ratio improved to 0.96 . By further numerically removing tip/tilt, as is typically done via a separate control loop, the time-averaged Strehl ratio approaches 0.99 , an overall increase of approximately $16.5 \%$. Figure 17 shows Strehl ratio versus time after both $\mathrm{AO}$ corrections have been applied and tip/tilt has been removed. It should be noted that time-averaging is computed in each of these numerical studies after phase-locking occurs. In this particular case, the AO corrections become phase-locked with the shear layer's emerging wavefront approximately $0.023 \mathrm{~s}$ after the controller is turned on.

Upper and lower Mach numbers were increased to approximately 0.7 and 0.2 , respectively for the second case reported here. This corresponds to an overall increase in the simulated shear layer's convective velocity from the first case discussed previously. In this case, forcing was applied at $1200 \mathrm{~Hz}$, producing a regularized region centered about $0.35 \mathrm{~m}$ downstream from the shear layer's origin. Figure 18 shows six consecutive wavefronts representing OPD error before forcing and $\mathrm{AO}$ corrections are applied. The time-averaged $\mathrm{OPD}_{\mathrm{rms}}$ error is approximately $0.068 \mu \mathrm{m}$. After regularizing the large-scale structures through forcing and applying $\mathrm{AO}$ corrections using the simulated PLL controller, the $\mathrm{OPD}_{\mathrm{rms}}$ error is reduced to approximately $0.038 \mu \mathrm{m}$. Instantaneous consecutive wavefront error post-AO corrections are shown in Fig. 19.

Strehl ratios were also computed before and after AO corrections were applied over a $0.035 \mathrm{~s}$ time interval. Figure 20 shows the Strehl ratio versus time calculated from the numerical results shown in Fig. 18. Figure 21 shows the Strehl ratio versus time for the forced shear layer while AO corrections are being applied based on the numerical wavefronts from Fig. 19. The time-averaged Strehl ratio increases from approximately $0 . \overline{63}$ to 0.96 once tip/tilt is removed 

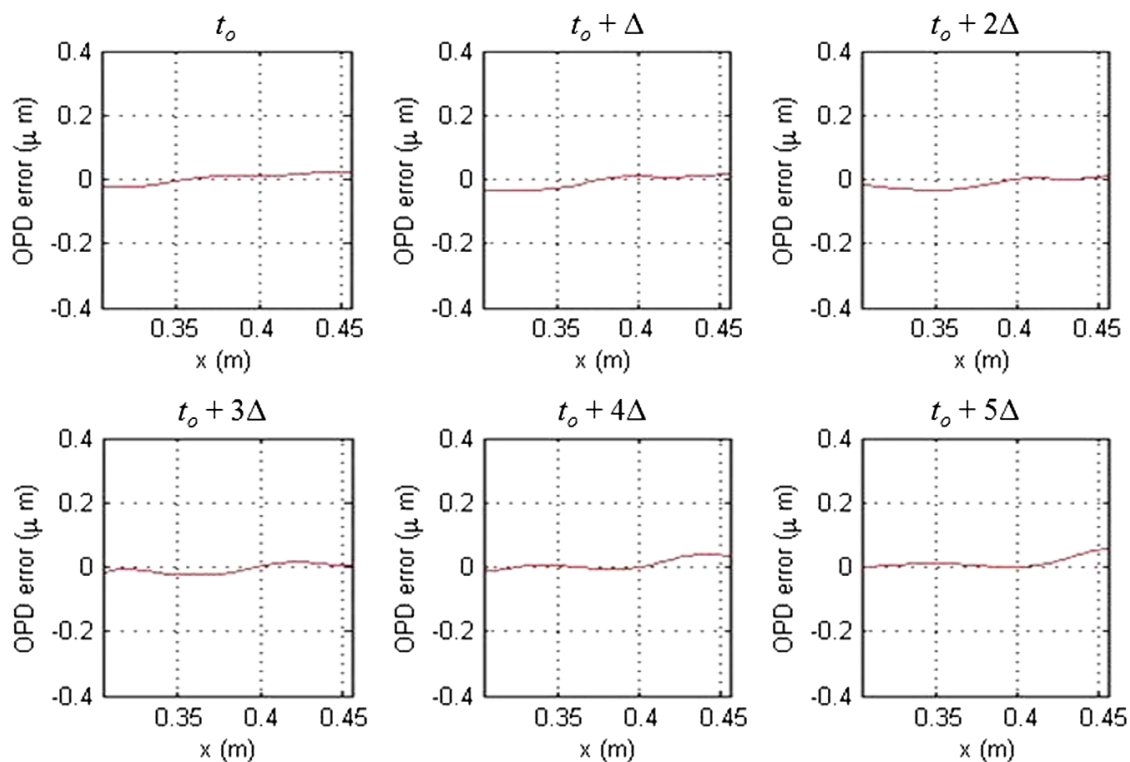

Fig. 15 Successive numerical wavefronts for an $1100 \mathrm{~Hz}$ forced Mach 0.55/0.17 shear layer with AO corrections.

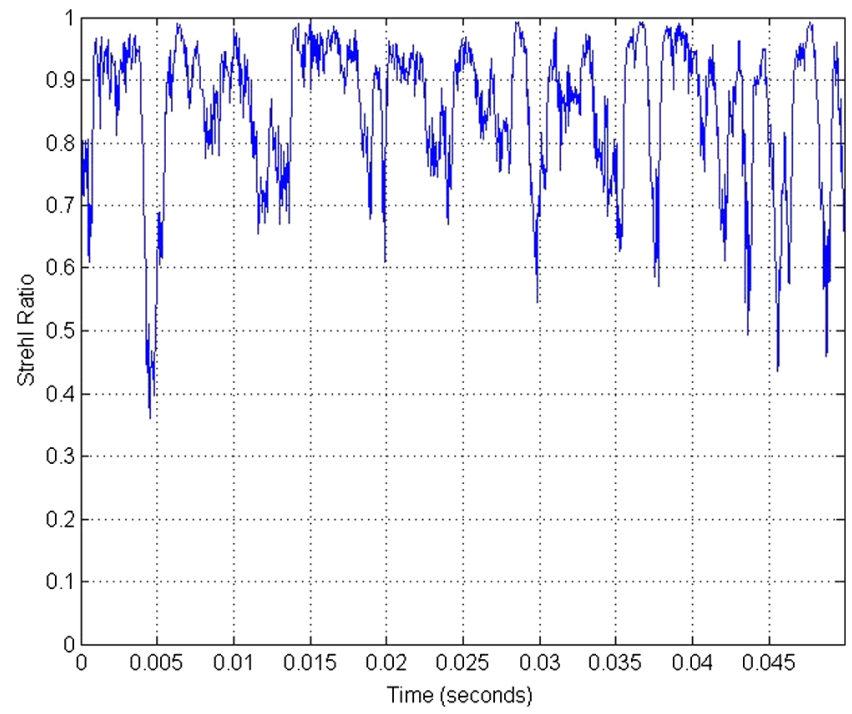

Fig. 16 Strehl ratio versus time for a Mach $0.55 / 0.17$ unforced shear layer.

and phase-locking occurs. The instantaneous Strehl ratio also no longer drops below approximately 0.75 , a significant improvement from the unforced, uncontrolled case.

The third shear layer case reported in this paper was chosen to demonstrate the effects resulting when a subharmonic becomes more dominant upon forcing. In this case, a shear layer was simulated with upper and lower Mach numbers of approximately 0.79 and 0.11 , respectively. Figure 22 shows the Strehl ratio versus time for the unforced case before forcing and AO corrections. After analyzing the natural optical frequencies present within the unforced shear layer [9], a forcing frequency of $700 \mathrm{~Hz}$ was selected to regularize the flow approximately $0.38 \mathrm{~m}$ downstream from the splitter plate. Figure $\underline{23}$ shows the Strehl ratio versus time for the $700 \mathrm{~Hz}$ forced shear layer while applying simulated AO corrections using the PLL controller described in this paper. In this case, forcing the shear layer did generate a more regular, periodic region within the flowfield; however, the emerging wavefront from this region is no longer dominated by the fundamental forcing frequency alone, but it is influenced significantly by its subharmonic. The PLL controller is still able to lock onto the shear layer's regularized jitter signal, but since the estimated conjugate correction is based upon a single

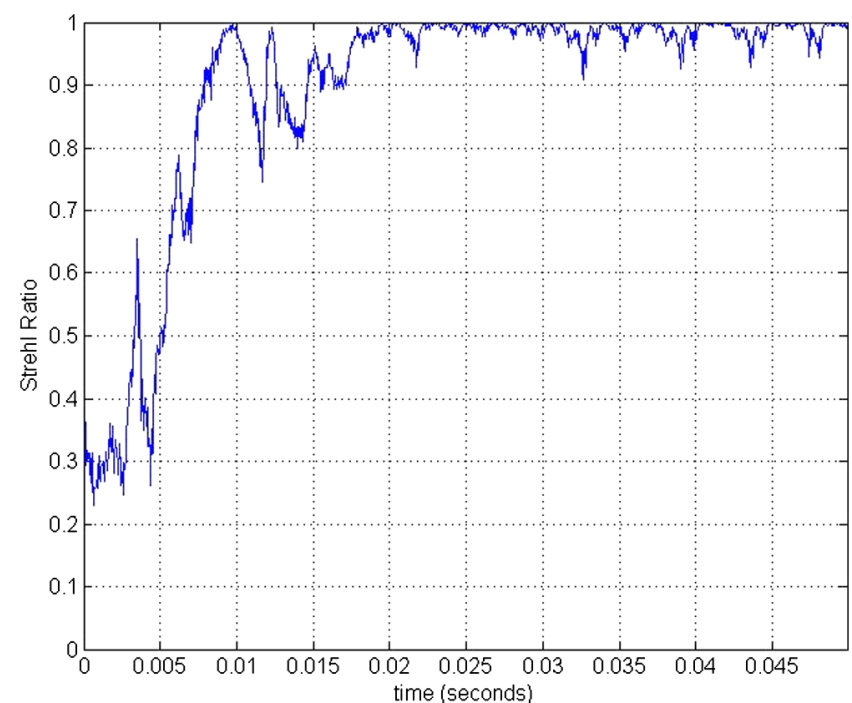

Fig. 17 Strehl ratio versus time for an $1100 \mathrm{~Hz}$ forced Mach 0.55/0.17 shear layer with $\mathrm{AO}$ corrections.

frequency, there exists a repetitive drop in the Strehl ratio, seen in Fig. 23, related to the subharmonic. The time-averaged Strehl ratio improves from approximately 0.13 in the unforced case to approximately 0.64 after forcing and $\mathrm{AO}$ corrections are applied with tip/tilt removed.

While the results shown in Figs. 21 and 22 do demonstrate a significant improvement, this type of situation requires a corrective form that includes the subharmonic in order to further improve the Strehl ratio and system performance. When doing so, the phase lag between the fundamental and subharmonic must be determined and phase-locking may require some slight changes to the PLL design. These variations to the current PLL controller are the subject of ongoing research at the University of Notre Dame. The following section describes the circuitry components and layout of the analog controller developed for this research effort.

\section{Controller Circuitry}

The AO controller described throughout this paper was designed to apply real-time $\mathrm{AO}$ corrections to an optical beam propagating through a regularized region of a high-speed free shear layer. A region 

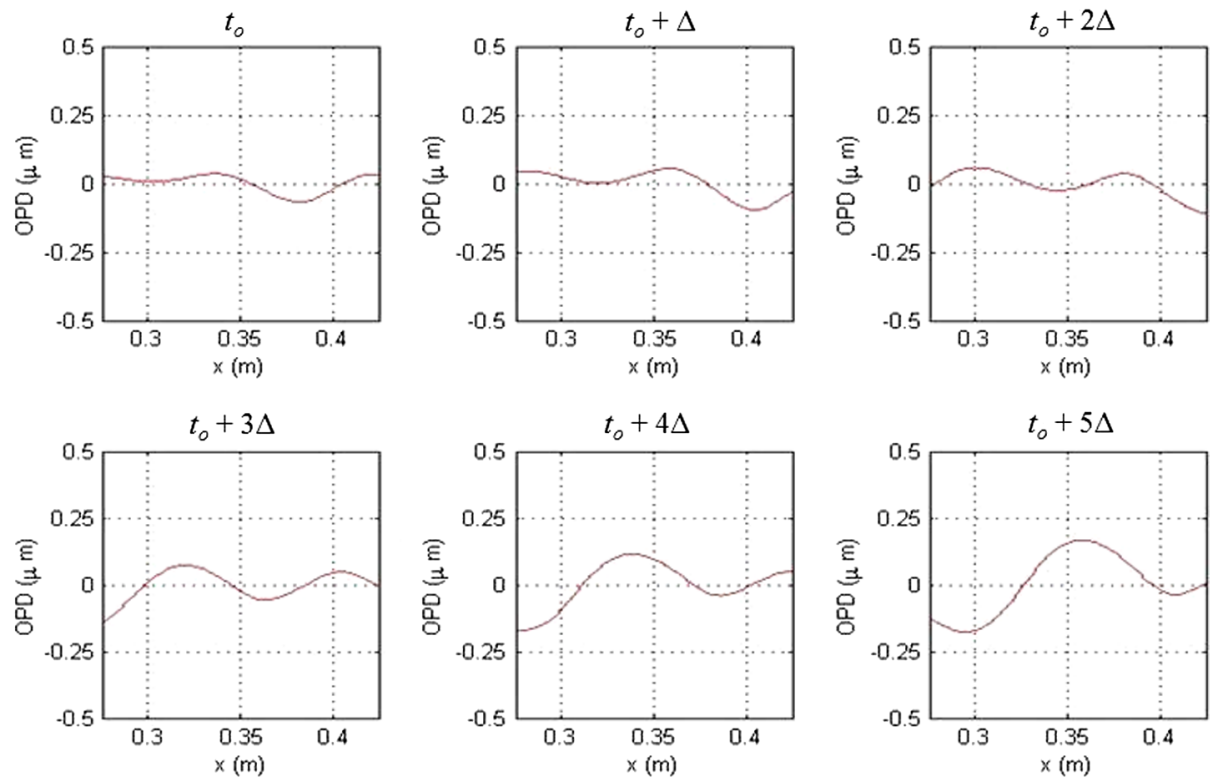

Fig. 18 Successive numerical wavefronts for a Mach 0.7/0.2 unforced shear layer.
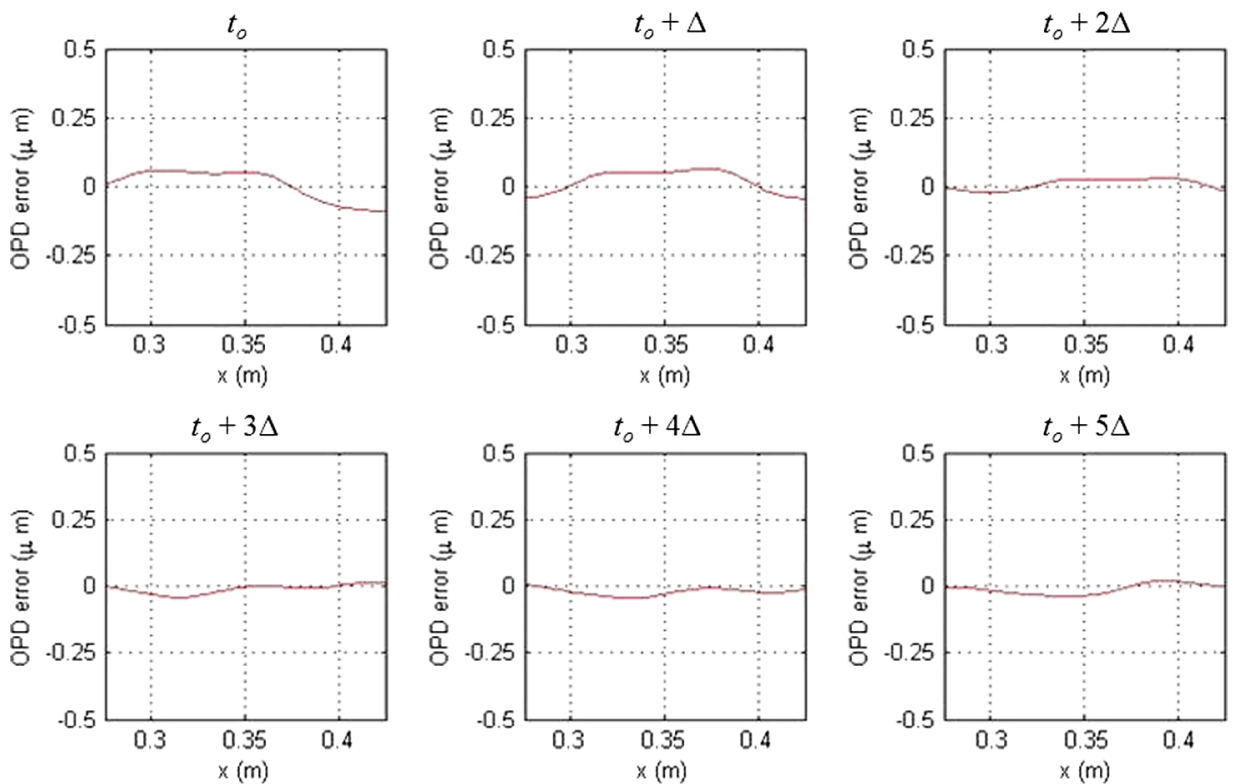

Fig. 19 Successive numerical wavefronts for a $1200 \mathrm{~Hz}$ forced Mach $0.7 / 0.2$ shear layer with AO corrections.

of regularized, more predictable large-scale structures and optical wavefronts must first be created by applying flow control to the shear layer itself [9-11,16,17]. Recent wind-tunnel experiments conducted at the University of Notre Dame have demonstrated the ability to generate such a region in a high-speed shear layer using mechanical forcing applied at the splitter plate [11]. Once this is accomplished, the alternative AO controller synchronizes an estimated wavefront correction, based upon the single forcing frequency, with the shear layer's actual emerging wavefront. The controller is designed to perform amplitude adjustments to the corrective waveform in addition to achieving phase-locking between wavefronts. The controller consists of two main components: the PLL circuit and an amplitude estimator circuit. The PLL circuit can further be separated into its phase detector, loop filter, and voltage-controlled oscillator (or waveform generator) subcomponents.

A jitter signal emerging from the regularized region of the forced shear layer acts as the PLL's reference source. The signal is initially scaled, using a four-quadrant multiplier/divider chip (AD734), generating an output for which the amplitude is approximately $2 \mathrm{~V}$.
Phase detection is achieved through a simple multiplication and lowpass filter combination; the scaled output is multiplied with the waveform generators output (PLL output) producing a mixed signal consisting of a double harmonic and a dc portion. These two signals are mixed using a four-quadrant multiplication chip (AD633). The mixed signal is passed through an active low-pass filter comprising a low-noise operational amplifier (NE5534A), two resistors, and a capacitor. The filter is designed to attenuate the double harmonic and isolate the dc component; the dc signal contains the important phaselag information necessary to determine the phase difference between jitter signals. Next, a loop filter, consisting of a low-noise operational amplifier (NE5534A) in combination with a capacitor and two resistors, acts as a regulator ensuring zero-tracking error for both step and ramp changes in phase while maintaining closed-loop system stability. The loop filter's output represents the phase adjustment necessary to begin synchronizing the two jitter signals. This voltage signal is summed, using an operational amplifier (NE5534A) summation circuit, with the waveform generator (NTE864) FM Bias output signal (the voltage required for the waveform generator to 


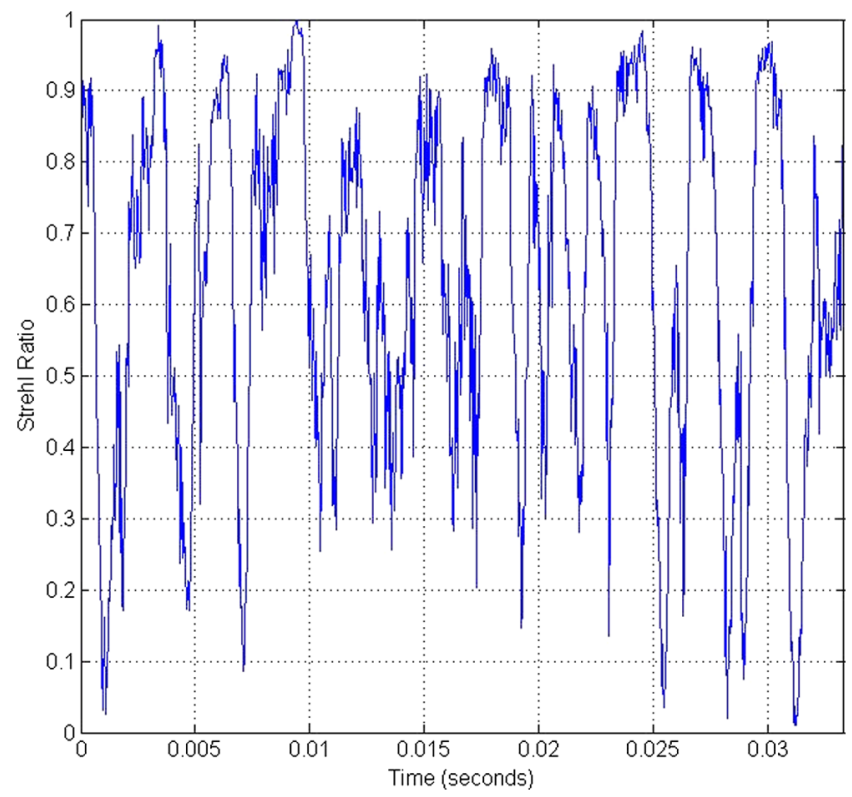

Fig. 20 Strehl ratio versus time for a Mach 0.7/0.2 unforced shear layer.

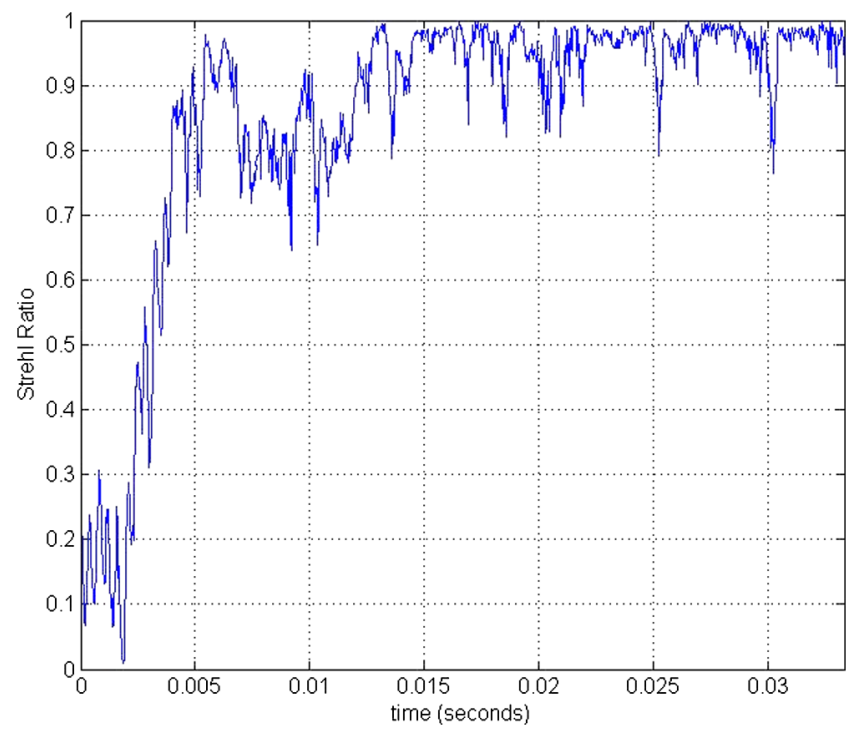

Fig. 21 Strehl ratio versus time for a $1200 \mathrm{~Hz}$ forced Mach 0.7/0.2 shear layer with AO corrections.

operate at its center operating frequency where FM refers to 'frequency modulated'). Finally, the signal is inverted (operational amplifier inverting circuit; NE5534A) producing an output that serves as the waveform generators FM sweep input. The waveform generator (NTE864) produces a sinusoidal output based upon its center frequency and the FM sweep input. The center frequency is selected externally using a combination of resistors and a capacitor, while frequency modulation is adjusted by an external voltage signal called the FM sweep input. The phase-locked output signal from the waveform generator is scaled to unity (operational amplifier inverter amplification circuit; NE5534A) before closing the PLL's feedback loop.

A separate control loop is used to estimate the appropriate amplitude for the conjugate correction. The amplitude of the shear layer's jitter signal is approximated using a rms-to-dc converter chip (AD536A). The chip produces a dc output equivalent to the true root mean square of the incoming ac signal; given a sinusoidal input, this equates to approximately 0.707 times the signal's amplitude. Consequently, the signal must be scaled (using an operational amplifier inverting amplification circuit; NE5534A) by approximately 1.414

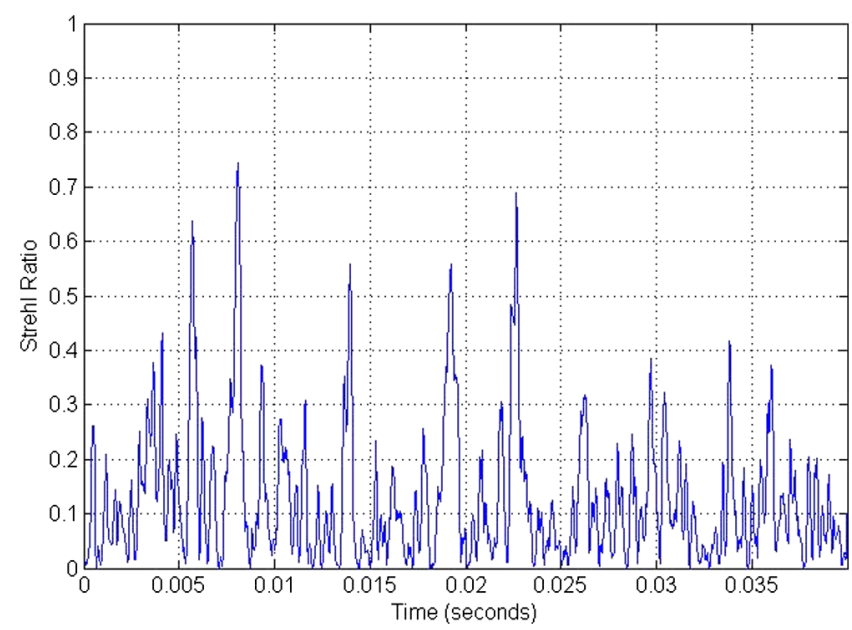

Fig. 22 Strehl ratio versus time for a Mach $0.79 / 0.11$ unforced shear layer.

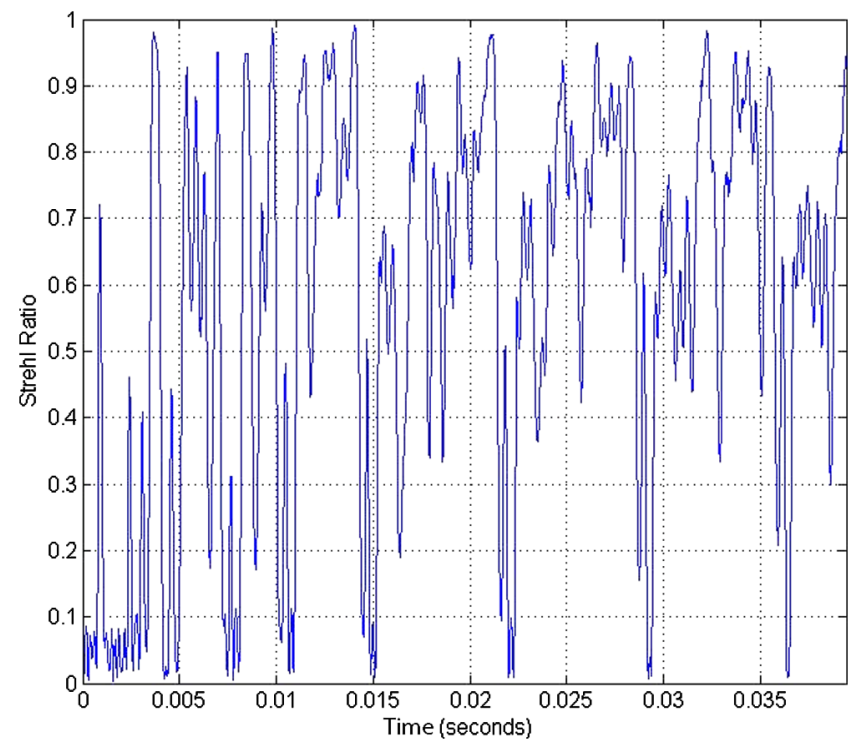

Fig. 23 Strehl ratio versus time for a $700 \mathrm{~Hz}$ forced Mach 0.79/0.11 shear layer with AO corrections.

generating an estimate of the original signal's amplitude. The estimated amplitude is then multiplied with the waveform generator's scaled output using another four-quadrant multiplier chip (AD633). The resulting output represents a sinusoidal jitter signal for which the phase and amplitude are being continually adjusted to synchronize with the shear layer's actual emerging jitter signal. Figure 24 displays a schematic of the overall AO controller circuitry.

The final step in the AO correction process will be to construct the deformable mirror's wavefront correction based on the phase-locked amplitude adjusted PLL jitter signal. Once the PLL output is scaled by the appropriate correction factor, accounting for the relationship between the jitter signal and optical wavefront, as well as the deformable mirror's voltage to displacement factor, the signal will be used to create a two-dimensional conjugate correction varying in real time. Continued research is being conducted to develop the best method of constructing this waveform. One possibility is to use a series of operational amplifier phase-lag circuits designed to apply the appropriate phase lag or lead to the original signal, thereby producing seven equally phase-shifted signals used to control the seven rows of actuators on the deformable mirror.

It should be noted that an additional 90 deg phase-lag circuit was used in the jitter signal experiments reported in the following section; however, this phase-lag portion is not part of the final PLL design. The PLL controller described in this paper will ultimately be used to 


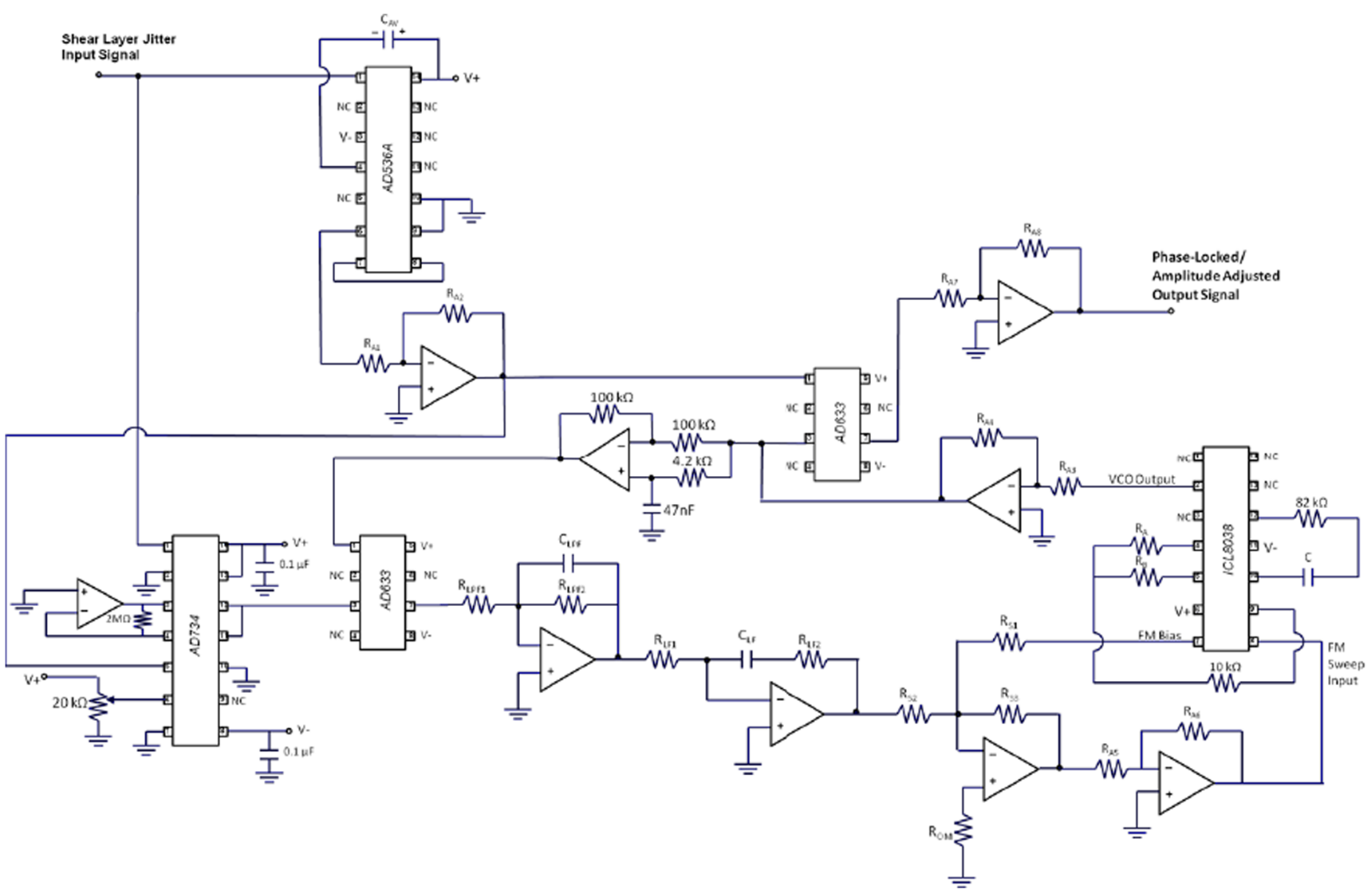

Fig. 24 Schematic showing the overall circuitry for the alternative AO PLL controller.

perform real-time corrections to a shear layer's aberrating wavefront. As such, the PLL's output jitter signal will be used to control the deformable mirror's conjugate correction, or wavefront. Since a jitter signal is related to the optical wavefront through a derivative, these two signals must be locked 90 deg out of phase. But, in order to create two phase-locked jitter signals in which synchronization could be more easily seen visually, a 90 deg phase-lag circuit was added for this purpose alone.

\section{Jitter Signal Experimental Results}

A series of jitter signal experiments were conducted on a prototype PLL circuit board. The purpose of these tests was to study the controller's phase-locking and amplitude adjustment capabilities. The results reported here are for a controller for which the center operating frequency is approximately $850 \mathrm{~Hz}$. Experimental testing was conducted using a GW dual tracking laboratory dc power supply set to $\pm 12 \mathrm{~V}$ operating in series. A function generator was used to simulate the shear layer's regularized jitter signal by providing a sinusoidal reference input with variable input frequency and amplitude. The input amplitude varied between 0.5 and $5 \mathrm{~V}$, while the input frequency varied between 500 and $1500 \mathrm{~Hz}$. A two-channel oscilloscope was used to monitor the reference input in conjunction with the PLL's phase-locked output signal. A data acquisition system was also used to record several signals during testing, including the shear layer jitter input signal (function generator signal), the scaled shear layer jitter signal, the phase-locked/amplitude adjusted PLL output signal, the scaled PLL output signal, and the amplitude estimation signal. The sixth channel from the data acquisition system was connected to the power supply's positive supply voltage, providing a reference signal showing when the experiment began. Data were recorded at a rate of $200 \mathrm{kHz}$ over a period of $5 \mathrm{~s}$ for each test.

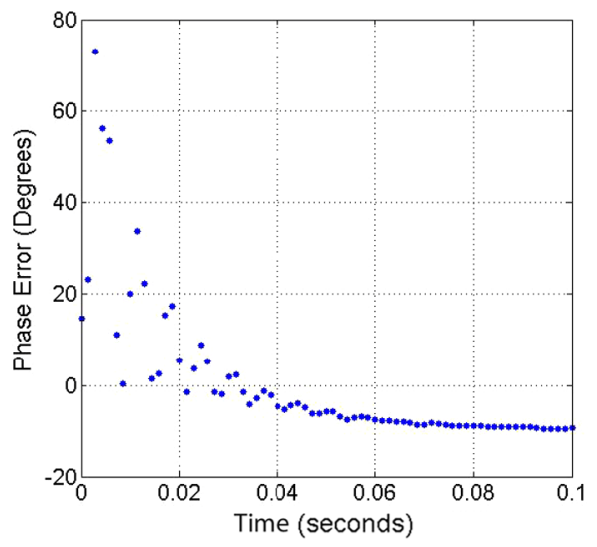

a)

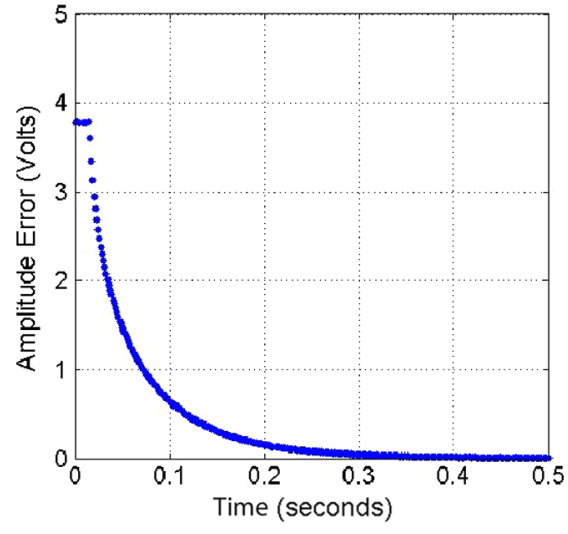

b)

Fig. 25 a) Phase error and b) amplitude error versus time for the PLL controller's response given a $700 \mathrm{~Hz}$ reference. 
The following figures show the phase-locking and amplitude adjustment results from a series of experimental tests. Consistent phase-locking results were obtained for input frequencies ranging from approximately 700 to $1200 \mathrm{~Hz}$, representing the PLL's capture range. Amplitude estimations settle to their final value within $0.5 \mathrm{~s}$ or less. Figures $25 \mathrm{a}$ and $25 \mathrm{~b}$ show phase error versus time, and amplitude error versus time, respectively, given an input frequency of $700 \mathrm{~Hz}$ and an input amplitude of $4 \mathrm{~V}$. Similar results to those shown in Fig. 25 were recorded at this frequency for amplitudes ranging between 0.5 and $5 \mathrm{~V}$. Note that there exists approximately $10 \mathrm{deg}$ of steady-state phase error in Fig. 25a. This is caused primarily by the 90 deg phase-shift circuit, which is designed based upon the center operating frequency of $850 \mathrm{~Hz}$. Therefore, a small amount of steadystate error is induced at frequencies other than $850 \mathrm{~Hz}$, which increases as the discrepancy between input frequency and the phaseshift circuit design frequency increases. However, the actual PLL controller used to construct wavefront corrections for a high-speed shear layer will not include this phase-shift circuit. Therefore, its associated steady-state error will also be omitted.

The second set of tests was conducted given a function generator input frequency of $800 \mathrm{~Hz}$ and an amplitude of $1 \mathrm{~V}$. In this case (results shown in Fig. 26), phase-locking occurs in approximately $0.05 \mathrm{~s}$, with very little steady-state error, and the signal amplitude is closely estimated in approximately $0.5 \mathrm{~s}$. Figure 27 shows the phase

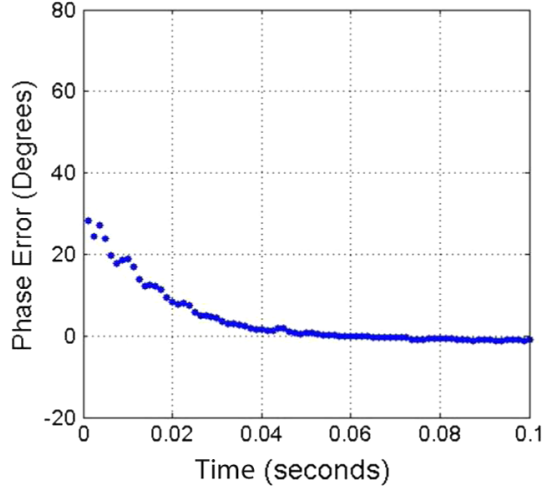

a)

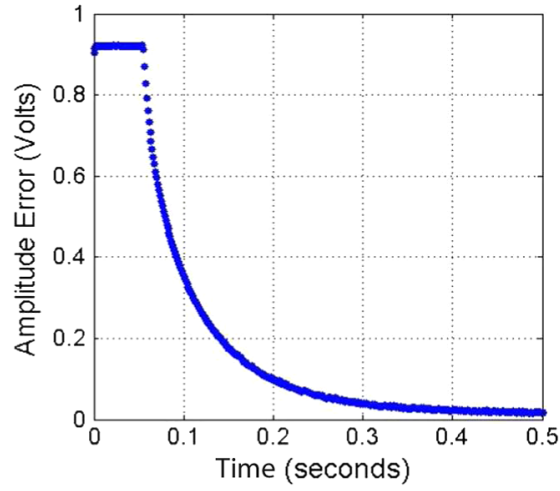

b)

Fig. 26 a) Phase error and b) amplitude error versus time for the PLL controller's response given an $800 \mathrm{~Hz}$ reference.

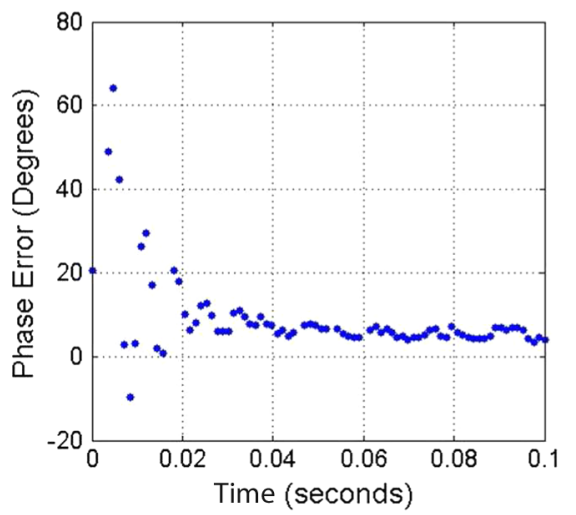

a)

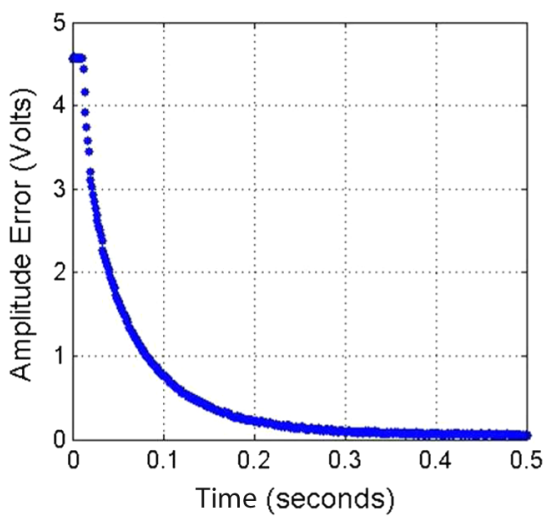

b)

Fig. 27 a) Phase error and b) amplitude error versus time for the PLL controller's response given a $900 \mathrm{~Hz}$ reference.

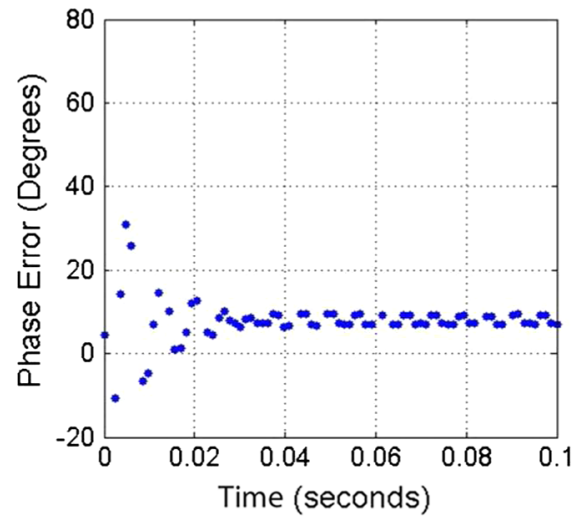

a)

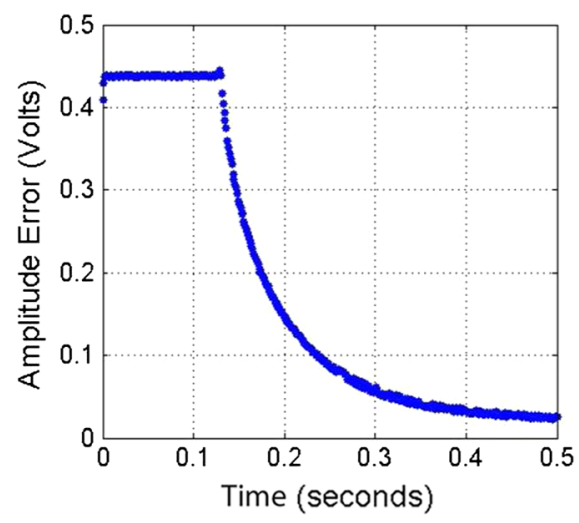

b)

Fig. 28 a) Phase error and b) amplitude error versus time for the PLL controller's response given a $1000 \mathrm{~Hz}$ reference. 


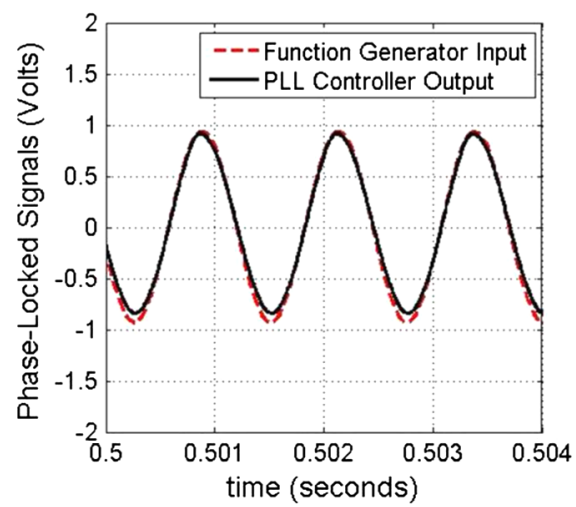

a)

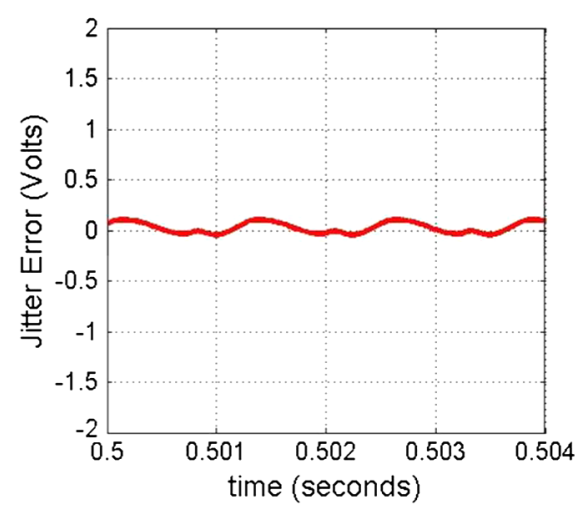

b)

Fig. 29 a) Input/output signals and b) error versus time for the PLL experimental test given an $800 \mathrm{~Hz}$ reference.

error and amplitude error results given an input frequency of $900 \mathrm{~Hz}$ and input amplitude of $5 \mathrm{~V}$. Finally, Fig. 28 shows the same set of results given an input reference signal for which the frequency and amplitude are $1000 \mathrm{~Hz}$ and $0.5 \mathrm{~V}$, respectively. At each input frequency shown in Figs. 25-28, similar results were obtained for input amplitudes ranging from 0.5 to $5 \mathrm{~V}$.

A snapshot of the actual jitter signals, the function generator reference signal, and the PLL's phase-locked signal is shown in Fig. 29a, given an input frequency and amplitude of $800 \mathrm{~Hz}$ and $1 \mathrm{~V}$, respectively. The associated phase error between the two signals is also shown in Fig. 29b over this time interval. These figures are meant to provide a more visual representation of the PLL controller's synchronization results.

\section{Conclusions}

Due to the bandwidth limitations inhibiting current AO systems in high-speed high-frequency cases, an alternative approach to overcoming this $\mathrm{AO}$ problem was proposed and investigated. This alternative AO approach combines flow control with a PLL control strategy in order to synchronize an estimated conjugate correction with a forced shear layer's regularized wavefront. It has been shown that a high-speed free shear layer's large-scale structures and their associated optical wavefronts may be made more regular and more predictable by mechanically forcing its origin [9-11]. A PLL controller was designed to perform real-time phase and amplitude adjustments to an estimated conjugate correction with the future goal of using this waveform to control a deformable mirror in applying real-time high-speed shear layer AO corrections. The controller was constructed on a prototype circuit board. The design process and circuitry layout has been reported in this paper. Initial experimental testing has been conducted using a function generator to simulate the forced shear layer's regularized jitter signal. The PLL controller presented in this paper was designed with a center operating frequency of approximately $850 \mathrm{~Hz}$ (which can be varied easily by swapping out a couple resistors and a capacitor). The controller demonstrated a good capture range between approximately 700 and $1200 \mathrm{~Hz}$. Phase locking was achieved in $0.1 \mathrm{~s}$ or less, while the amplitude estimation was attained in $0.5 \mathrm{~s}$ or less. The next phase of this research is to perform real-time jitter corrections to a smallaperture beam propagating through a forced shear layer using the PLL controller presented here to control a tip/tilt mirror. Finally, the controller will be used to control a deformable mirror in a high-speed shear layer $\mathrm{AO}$ wavefront correction experiment. This alternative $\mathrm{AO}$ approach represents an innovative way of overcoming bandwidth limitations faced by current systems.

\section{Acknowledgment}

These efforts were sponsored by the U.S. Air Force Office of Scientific Research, Air Force Material Command, under grant number FA9550-06-1-0160. The U.S. Government is authorized to reproduce and distribute reprints for governmental purposes notwithstanding any copyright notation thereon.

\section{References}

[1] Tyson, R. K., Principles of Adaptive Optics, 2nd ed., Academic Press, Chestnut Hill, MA, 1991, pp. 1-5, 25-36, 53-66, 117-210.

[2] Tyson, R. K., "Adaptive Optics Compensation of Atmospheric Turbulence: The Past, the Present, and the Future," Atmospheric Propagation and Remote Sensing III Conference, Vol. 2222, SPIE, Bellingham, WA, April 1994, pp. 404-412.

[3] Lloyd-Hart, M., "Taking the Twinkle Out of Starlight," IEEE Spectrum, Vol. 40, No. 12, Dec. 2003, pp. 22-29. doi:10.1109/MSPEC.2003.1249975

[4] Beckers, J. M., "Adaptive Optics for Astronomy: Principles, Performance, and Applications," Annual Review of Astronomy and Astrophysics, Vol. 31, Sept. 1993, pp. 13-62. doi:10.1146/annurev.aa.31.090193.000305

[5] Jumper, E. J., and Fitzgerald, E. J., "Recent Advances in Aero-Optics," Progress in Aerospace Sciences, Vol. 37, No. 3, 2001, pp. 299-339. doi:10.1016/S0376-0421(01)00008-2

[6] Kyrazis, D. T., "Optical Degradation by Turbulent Free Shear Layers," Proceedings of SPIE, Vol. 2005, Dec. 1993, pp. 170-181. doi:10.1117/12.163700

[7] Duffin, D. A., "Feed-Forward Adaptive-Optic Correction of AeroOptical Aberrations Caused by a Two-Dimensional Heated Jet," AIAA Paper 2005-4776, June 2005.

[8] Cicchiello, J. M., and Jumper, E. J., "Far-Field Optical Degradation Due to Near-Field Transmission Through a Turbulent Heated Jet," Applied Optics, Vol. 36, No. 25, 1997, pp. 6441-6452. doi:10.1364/AO.36.006441

[9] Nightingale, A. M., Gordeyev, S., and Jumper, E. J., "Optical Characterization of a Simulated Weakly Compressible Shear Layer: Unforced and Forced," AIAA Journal, Vol. 47, No. 10, Oct. 2009, pp. $2298-2305$. doi: $10.2514 / 1.34244$

[10] Rennie, R. M., Duffin, D. A., and Jumper, E. J., "Characterization and Aero-Optic Correction of a Forced Two-Dimensional, WeaklyCompressible Shear layer," AIAA Journal, Vol. 46, No. 11, Nov. 2008, pp. 2787-2795. doi:10.2514/1.35290

[11] Rennie, R. M., Siegenthaler, J. P., and Jumper, E. J., "Forcing of a TwoDimensional, Weakly-Compressible Subsonic Free Shear Layer," AIAA Paper 2006-0561, Jan. 2006.

[12] Brown, G. L., and Roshko, A., "On Density Effects and Large Structure in Turbulent Mixing Layers," Journal of Fluid Mechanics, Vol. 64, No. 4,1974 , pp. $775-816$. doi:10.1017/S002211207400190X

[13] Brown, G. L., "The Entrainment and Large-Scale Structure in Turbulent Mixing Layers," Fifth Australian Conference on Hydraulics and Fluid Mechanics, Vols. 1-2, University of Canterbury, Christchurch, New Zealand, 1974, pp. 352-359.

[14] Winant, C. D., and Browand, F. K., "Vortex Pairing: the Mechanism of Turbulent Mixing Layer Growth at Moderate Reynolds Number," Journal of Fluid Mechanics, Vol. 63, No. 2, 1974, pp. 237-255. doi:10.1017/S0022112074001121

[15] Fitzgerald, E. J., and Jumper, E. J., "The Optical Distortion Mechanism in a Nearly Incompressible Free Shear Layer," Journal of Fluid 
Mechanics, Vol. 512, Aug. 2004, pp. 153-189.

doi: $10.1017 / \mathrm{S} 0022112004009553$

[16] Ho, C.-M., and Huerre, P., "Perturbed Free Shear Layers," Annual Reviews of Fluid Mechanics, Vol. 16, Jan. 1984, pp. 365-424. doi:10.1146/annurev.fl.16.010184.002053

[17] Wygnanski, I., and Oster, D., "The Forced Mixing Layer Between Parallel Streams," Journal of Fluid Mechanics, Vol. 123, Oct. 1982, pp. 91-130. doi:10.1017/S0022112082002961

[18] Abramovitch, D., "Phase-Locked Loops: A Control Centric Tutorial," Proceedings of the American Control Conference, Vol. 1, IEEE, Communications \& Optics Research Lab., Agilent Labs., Palo Alto, CA, May 2002, pp. 1-15.
[19] Fitzgerald, E. J., Siegenthaler, J. P., and Jumper, E. J., "Optical Characterization of the Notre Dame Compressible Shear-Layer Facility," AIAA Paper 2002-2274, May 2002.

[20] Chouinard, M., Asghar, A., Kirk, J. F., Siegenthaler, J. P., and Jumper, E. J., "An Experimental Verification of the Weakly-Compressible Model," AIAA Paper 2002-0352, Jan. 2002.

M. Glauser Associate Editor 